\title{
Cowden syndrome: gastrointestinal manifestations of an infrequent polyposis
}

A 58-year-old man, with a history of total thyroidectomy for papillary carcinoma in 2012, presented with rectal bleeding for the last 4 years, without other digestive symptoms or weigh lost. His physical examination revealed macrocephaly (63-cm circumference), skin with multiple verrucous papules of $1-3 \mathrm{~mm}$ on the face and dorsum of his hands (which histologically were trichilemmomas), papules in the oral mucosa (hamartomatous papillomas), and a furrowed tongue. The rest of his physical examination and laboratory test results were normal. Colonoscopy showed more than 50 sessile hamartomatous polyps of 2-5 mm in the rectum and sigmoid colon. Upper

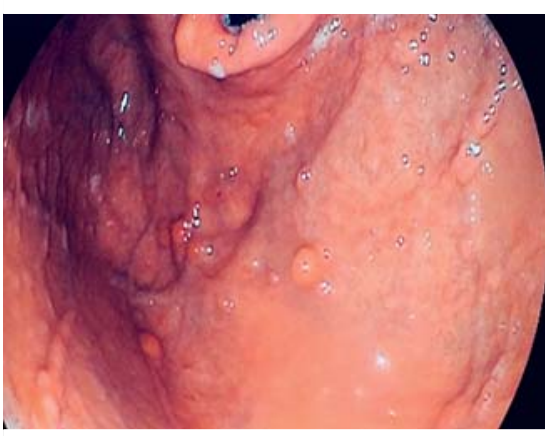

- Fig. 1 Endoscopic view showing sessile gastric polyps.

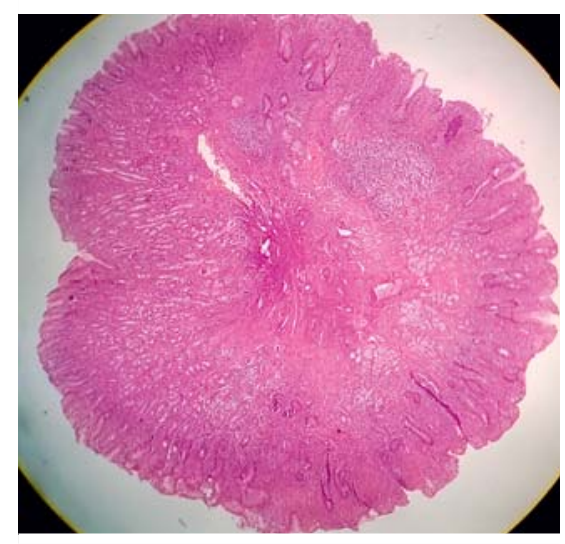

- Fig. 2 Histology of a resected gastric polyp showing a hamartoma. gastrointestinal endoscopy showed flat whitish lesions of 3-7 mm in the distal esophagus (acanthosis glycogen). In the stomach, multiple sessile polyps of 3-5 mm ( Fig. 1) were resected and histology revealed these to be hamartomas ( $\mathbf{F i g . 2}$ ). In the second portion of the duodenum, a few sessile polyps of 4-5 mm were resected (histology revealed normal mucosa).

Small-bowel capsule endoscopy revealed multiple polyps of $1-3 \mathrm{~mm}$ in the duode- num, proximal jejunum ( $\triangleright$ Fig.3), and distal ileum ( $\triangleright$ Video 1 ).

Cowden syndrome is a rare entity related to the PTEN gene. It is characterized by the presence of hamartomatous lesions in any location. The diagnostic criteria (International Cowden Consortium criteria) include major and minor criteria ( $\triangleright$ Table 1). Major criteria include: multiple gastrointestinal hamartomas or ganglioneuromas, macrocephaly, macular pigmentation of glans penis, mucocu-
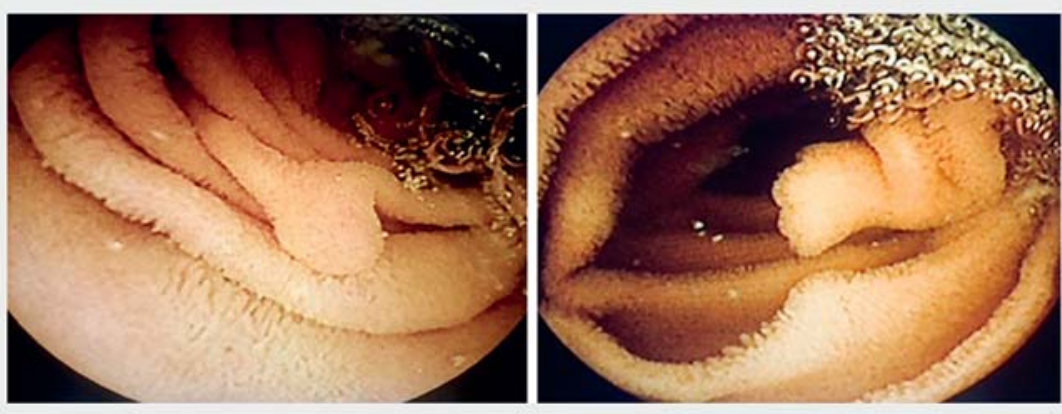

- Fig. 3 Small-bowel capsule endoscopy images showing jejunal polyps.
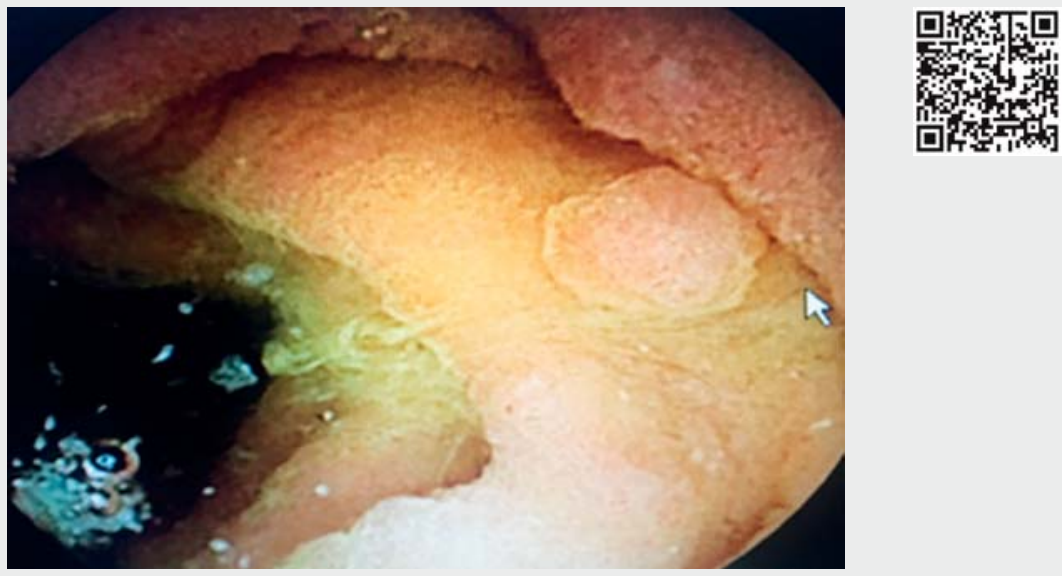

$\checkmark$ Video 1 Small-bowel capsule endoscopy revealed multiple sessile polyps of $1-3 \mathrm{~mm}$ in the duodenum, proximal jejunum, and distal ileum. 
- Table 1 Diagnostic criteria for Cowden syndrome (International Cowden Consortium criteria updated by the National Comprehensive Cancer Network 2018).

\begin{tabular}{|c|c|}
\hline Major criteria & Minor criteria \\
\hline $\begin{array}{l}\text { Breast cancer } \\
\text { Endometrial cancer } \\
\text { Follicular thyroid cancer } \\
\text { Multiple gastrointestinal hamartomas or } \\
\text { ganglioneuromas } \\
\text { Macrocephaly }(58 \mathrm{~cm} \text { in adult women, } 60 \mathrm{~cm} \\
\text { in adult men) } \\
\text { Macular pigmentation of glans penis } \\
\text { Mucocutaneous lesions: } \\
\text { - One biopsy-proven trichilemmoma } \\
\text { - Multiple palmoplantar keratoses } \\
\text { - Multifocal or extensive oral mucosal } \\
\text { - Mapillomatosis }\end{array}$ & $\begin{array}{l}\text { Autism spectrum disorder } \\
\text { Colon cancer } \\
\geq 3 \text { esophageal glycogenic acanthoses } \\
\text { Lipoma } \\
\text { Intellectual disability (IQ } \leq 75 \text { ) } \\
\text { Papillary or follicular variant of papillary } \\
\text { thy-roid cancer } \\
\text { Thyroid structural lesions (adenoma, nodules, } \\
\text { goiter) } \\
\text { Renal cell carcinoma } \\
\text { Single gastrointestinal hamartoma or gang- } \\
\text { lioneuroma } \\
\text { Testicular lipomatosis } \\
\text { Vascular anomalies (including multiple intra- } \\
\text { cranial developmental venous anomalies) }\end{array}$ \\
\hline \multicolumn{2}{|c|}{$\begin{array}{l}\text { Operational diagnosis in an individual (either of the following): } \\
\text { 1. Three or more major criteria, but one must include macrocephaly, Lhermitte-Duclos disease, } \\
\text { or Gl hamartomas } \\
\text { 2. Two major and three minor criteria }\end{array}$} \\
\hline \multicolumn{2}{|c|}{$\begin{array}{l}\text { Operational diagnosis in a family where one individual meets the revised clinical diagnostic } \\
\text { criteria for PTEN hamartoma tumor syndrome or has a PTEN mutation (one of the following): } \\
\text { 1. Any two major criteria with or without minor criteria } \\
\text { 2. One major and two minor criteria } \\
\text { 3. Three minor criteria }\end{array}$} \\
\hline
\end{tabular}

taneous lesions (trichilemmoma, palmoplantar keratoses, oral mucosal papillomatosis, and cutaneous facial papules), and breast, endometrial, or follicular thyroid cancer. Because of the risk of colorectal cancer, surveillance is recommended, with colonoscopy starting at age 35 or 10 years younger than the age of the index relative. Intervals should be based on the findings, with colonoscopy every 1-2 years if multiple polyps or adenomatous polyps are present, or every $3-5$ years if polyps are sparse or no polyps are found [1-4].

Endoscopy_UCTN_Code_CCL_1AC_2AC
Corresponding author

\section{Carolina Olano, MD}

Hospital de Clínicas, Av. Italia S/n CP, 11600, Montevideo, Uruguay

Fax: +598-2-4872572

carolinaolanouruguay@gmail.com

\section{References}

[1] Coriat R, Mozer M, Caux E et al. Endoscopic finding in Cowden syndrome. Endoscopy 2011; 43: $723-726$

[2] Shaco-Levy R, Jasperson KW, Martin KJ et al. Gastrointestinal polyposis in Cowden syndrome. Clin Gastroenterol 2017; 51: e60 e67

[3] Mester ], Eng C. Cowden syndrome: recognizing and managing a not-so-rare hereditary cancer syndrome. J Surg Oncol 2015; 111: $125-130$

[4] Saito K, Nomura E, Sasaki Y et al. Characteristics of small bowel polyps detected in Cowden syndrome by capsule endoscopy. Case Rep Gastrointest Med 2015; 2015: 1 - 4

\section{Bibliography}

DOI https://doi.org/10.1055/a-0885-9246

Published online: 16.5.2019

Endoscopy 2019; 51: E280-E281

(c) Georg Thieme Verlag KG

Stuttgart · New York

ISSN 0013-726X

The authors

Daniela Mendez ${ }^{1}$, Rodrigo Dorelo ${ }^{1}$, Martín Oricchio $^{1}$, Martha Otero ${ }^{2}$, Joaquin Berrueta ${ }^{1}$, Carolina Olano ${ }^{1}$

1 Clínica de Gastroenterología, Universidad de la República, Montevideo, Uruguay

2 Cátedra de Anatomía Patológica, Hospital de Clínicas, Montevideo, Uruguay

\section{ENDOSCOPY E-VIDEOS}

https://eref.thieme.de/e-videos

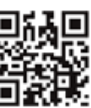
Endoscopy E-Videos is a free access online section, reporting on interesting cases and new

techniques in gastroenterological endoscopy. All papers include a high quality video and all contributions are freely accessible online.

This section has its own submission website at https://mc.manuscriptcentral.com/e-videos 